\title{
O GEOGEBRA COMO POTENCIALIZADOR NA APLICAÇÄO DE FUNÇÃO EXPONENCIAL
}

\section{GEOGEBRA AS A POTENTIALIZER IN THE APPLICATION OF EXPONENTIAL FUNCTION}

\author{
Roberta Borges Monteiro ${ }^{1}$ \\ ORCID iD: $\underline{0000-0002-1672-6450}$
}

Suyanne Rodrigues Alves Laranjeira ${ }^{2}$

ORCID iD: 0000-0003-4451-6124

Leyde Dayane Martinho de Andrade ${ }^{3}$

ORCID iD: $\underline{0000-0002-7558-1319}$

Jucicleia Gomes Ribeiro Neto 4

ORCID iD: $\underline{0000-0002-0370-4279}$

\section{RESUMO}

Este trabalho apresenta uma experiência transcorrida a partir de uma proposta de atividade na disciplina Tópicos Especiais de Cálculo do Mestrado Profissional em Ciências da Universidade Estadual de Roraima - UERR, no segundo semestre de 2016 com o objetivo de analisar como o software GeoGebra contribui para a resolução de um problema que propunha determinar como ocorre o processo de eliminação do medicamento Diazepam no organismo do paciente usuário. Evidenciouse que os recursos disponibilizados no software GeoGebra contribuem sim para a resolução de problemas que envolvem uma possibilidade de aplicação de Função Exponencial.

Palavras-chave: Resolução de Problemas. Software Geogebra. Função Exponencial.

\begin{abstract}
This paper presents an experience from an activity proposal in the Special Topics of Calculation course of the Professional Master in Science of the University "XXX", in the second semester of 2016 with the objective of analyzing how the GeoGebra software contributes to the resolution of a problem that proposed to determine how the process of elimination of the drug Diazepam occurs in the body of
\end{abstract}

\footnotetext{
${ }^{1}$ Mestranda em Ensino de Ciências pela Universidade Estadual de Roraima (UERR). Professora de Educação Básica da Prefeitura de Boa Vista (PMBV), Boa Vista, Roraima, Brasil. Endereço para correspondência: Rua: Mauro Campelo, 440, Joquei Clube, Boa Vista, Roraima, Brasil, CEP: 69315185. E-mail: robertaborges4393@gmail.com.

${ }^{2}$ Mestranda em Ensino de Ciências pela Universidade Estadual de Roraima (UERR). Professora de Educação Básica da Prefeitura de Boa Vista (PMBV), Boa Vista, Roraima, Brasil. Endereço para correspondência: Rua: Caruaru, 510, Centenário, Boa Vista, Roraima, Brasil, CEP: 69312520. E-mail: suyanner8@ gmail.com.

${ }^{3}$ Mestranda em Ensino de Ciências pela Universidade Estadual de Roraima (UERR). Professora - secretaria de Educação do Amazônas (seduc-AM), Presidente Figueiredo, Amazonas, Brasil. Endereço para correspondência: Rua: Guariba 88, Galo da Serra I, Presidente Figueiredo, Amazonas, Brasil, CEP: 69735000. E-mail: mleydedayane@hotmail.com.

${ }^{4}$ Mestranda em Ensino de Ciências pela Universidade Estadual de Roraima (UERR). Endereço para correspondência: Rua: Helena Bezerra de Menezes, 452, Liberdade, Boa Vista, Roraima, Brasil, CEP: 69309079. E-mail: j.gomesribeiro@ hotmail.com.
} 
the user patient. It was evidenced that the resources available in GeoGebra software contribute to the resolution of problems involving the possibility of applying Exponential Function.

Keywords: Problem Solving. Geogebra Software. Exponential Function.

\section{INTRODUÇÃO}

Todo processo de construção constituída pelas civilizações para a invenção do número, no intuito de problematizar situações matemáticas, deu início a uma funcionalidade conceitual e prática de situações numéricas.

Para Michels (2009), o ensino da Matemática tem passado, ao longo dos anos, por sucessivas reformas na busca de meios mais práticos e significativos de alcançar a aplicação dos conhecimentos matemáticos.

Assim a matemática tem uma representatividade como papel importante na vida do cidadão, uma vez que proporciona a construção do conhecimento favorecendo a possibilidade do aluno pensar, refletir, agir e criar soluções no âmbito matemático, como afirma (PERIUS, 2012, p. 13).

Aliado ao uso de Tecnologias Digitais - TD, o ensino da matemática torna-se mais atrativo a percepção de conceitos e fundamentos e sua aplicabilidade na execução de cálculos e da leitura matemática.

Podendo colaborar para o desenvolvimento de novos conhecimentos e no desenvolvimento de linguagens, vindo a ser um canal facilitador para a estrutura do desenvolvimento do educando como parte colaborativa e de cidadania.

Neste contexto insere-se a disciplina de Tópicos de Cálculo do Programa de PósGraduação em Ensino de Ciências (PPGEC), da Universidade Estadual de Roraima - UERR, na qual surgiu uma proposta de elaborar uma situação problema sobre funções exponenciais, cujo processo de resolução ocorresse com a utilização do software GeoGebra.

Diante disto o objetivo deste artigo é fazer uma reflexão teórica sobre algumas repercussões da função exponencial associada a uma atividade como fonte de pesquisa acerca da atividade pedagógica executada em dados existentes na bula do medicamento Diazepam, através do seu tempo de absorção entre o pico e momento em que começa a decair, para isso o desenvolvimento das ações transcorridas no processo se deu a partir da Teoria da Assimilação das Ações Mentais por Etapa proposta por Galperin. 
Dessa forma, a situação problema ora apresentada guiou as ações desenvolvidas de modo que se obteve uma solução para a mesma. Assim, apresentam-se, a seguir, as ações realizadas e o resultado obtido.

\section{REFERENCIAIS TEÓRICOS}

\subsection{Tecnologias Digitais e sua Aplicabilidade no Ensino da Matemática}

As Tecnologias Digitais (TD) e a Resolução de Problemas tem sido um tema presente em diversos debates, considerando suas potencialidades e limitações no contexto atual de nossas escolas.

Percebe-se que a utilização das Tecnologias Digitais (TD) faz parte da nossa atual sociedade, pois, atualmente, nossa cultura está fincando suas raízes na rede de computadores, na Internet, nos aparatos tecnológicos que cada vez mais buscam a evolução técnica.

Na Lei de Diretrizes e Bases da Educação Nacional - LDBEN está prevista, dentre outros elementos para a formação básica do cidadão em nível de Ensino Fundamental, a compreensão da tecnologia e suas implicações na sociedade. Para o Ensino Médio, a LDBEN recomenda, no Artigo 35, Inciso IV, que sejam explorados os conhecimentos "científicotecnológicos dos processos produtivos, relacionando a teoria com a prática, no ensino de cada disciplina" (BRASIL, 2001).

$\mathrm{O}$ uso das tecnologias digitais na educação tem como base o meio acadêmico e o poder público. Pois, é através deles que se pode fundamentar o uso da TD, nas escolas

[...] pode contribuir de diferentes modos com o processo de ensino e aprendizagem da Matemática. [Pois a tecnologia] [...] pode se constituir em uma ferramenta de auxílio à compreensão do raciocínio do aluno, de suas dificuldades e compreensões, além de ser uma poderosa ferramenta na elaboração de atividades que favoreçam a aprendizagem (MUSSATO apud BITTAR, 2015, p. 59).

Observa-se neste aspecto, que o uso das TD com o objetivo de potencializar a produção do conhecimento matemático ainda é falho, pois, o professor ainda tem dificuldade no uso das TD em sala de aula como funcionalidade no processo de ensino e aprendizagem. De acordo com Mussato (2015), o diferencial do trabalho com TD está relacionado à forma como o professor utilizará as TD e não que essas sejam utilizadas como recursos reproducionistas. 
Atualmente, a utilização do software no ensino da matemática busca permitir uma dinamicidade e a facilitação da aprendizagem dos conteúdos. Freire (2016, p.37), fala que para a disciplina de matemática existem softwares (livres ou proprietários) que podem fornecer uma melhor visualização de gráficos, figuras geométricas inclusive em 3D.

Freire (2016, p.37), também, define o software GEOGEBRA, como ferramenta que permitem a construção de figuras geométricas a partir das propriedades que as definem. Este programa matemático pode ser utilizado no Ensino Fundamental, Médio e Superior, principalmente nas atividades de geometria, cálculo e álgebra.

Assim, há uma grande predisposição na educação para acelerar a utilização de computadores como recurso didático em sala de aula. $\mathrm{O}$ avanço tecnológico, esperado pelos educadores, provém de uma propensa melhora do desempenho do processo de ensino e aprendizagem.

\subsection{A Resolução de Problemas}

A resolução de problemas vem sendo discutida há muitos anos, como uma proposta metodológica que proporciona um melhor desempenho do aluno no processo de ensino e aprendizagem.

\footnotetext{
“A História da Matemática mostra que ela foi construída como respostas a perguntas provenientes de diferentes origens e contextos, motivadas por problemas de ordem prática (divisão de terras, cálculo de créditos), por problemas vinculados a outras ciências (Física, Astronomia), bem como por problemas relacionados a investigações internas à própria Matemática.” (PCN, 2001, p.42).
}

Geralmente, os problemas matemáticos são inseridos pelo professor posteriormente ao conceito ensinado. Na prática, em sala de aula é muito comum o ensino mecânico e de memorização, através de exercícios. Com isso, o aluno só entende como problema matemático, algo que ele consiga resolver através de cálculo e às vezes tentando adivinhar o algoritmo necessário. Assim de acordo com o PCN de Matemática (2001), a prática mais frequente consiste em ensinar um conceito, procedimento ou técnica e depois apresentar um problema para avaliar se os alunos são capazes de empregar o que lhes foi ensinado.

Dessa forma, o aluno passa simplesmente a compreender que cada problema é direcionado a uma única operação matemática.

A partir dessa perspectiva, percebe-se que a ideia de resolução de problemas vem sendo mal interpretada, pois muitos professores compreendem erroneamente o seu objetivo. 
O PCN de Matemática (2001) defende que essa proposta poderia ser resumida seguindo os princípios abaixo:

- “O ponto de partida da atividade matemática não é a definição, mas o problema $[\ldots] ”$.

- “O problema certamente não é um exercício em que o aluno aplica, de forma quase mecânica, uma fórmula ou um processo operatório [...]”.

- “Aproximações sucessivas ao conceito são construídas para resolver um certo tipo de problema; num outro momento, o aluno utiliza o que aprendeu para resolver outros $[\ldots] "$.

- “O aluno não constrói um conceito em resposta a um problema, mas constrói um campo de conceitos que toma sentido num campo de problemas $[\ldots]$ ”.

- “A resolução de problemas não é uma atividade para ser desenvolvida em paralelo ou como aplicação da aprendizagem, mas uma orientação da aprendizagem $[\ldots] "$.

Dessa maneira, ao interpretar o que se caracteriza como resolução de problemas, o professor passará a entender que para o aluno resolver um problema matemático é necessário que ele perceba a sua construção a partir de uma sequência de ações e operações para que se chegue a um resultado correto e com sentido.

Nessa perspectiva, é que a resolução de problemas pode ser vista como ponto de partida da atividade matemática em contrapartida à simples resolução de procedimentos e ao acúmulo de informações, uma vez que possibilita aos estudantes a mobilização dos conhecimentos e o gerenciamento das informações que estão ao seu alcance.

Dante (2000), também assinala o trabalho com resolução de problemas matemáticos como a principal forma de se alcançar os objetivos da Matemática em sala de aula, entre eles, o de "fazer o aluno pensar produtivamente". O autor ainda destaca que, mais do que nunca, precisamos de pessoas ativas e participantes, que deverão tomar decisões rápidas e, tanto quanto possível, precisas.

\section{MATERIAIS E MÉTODOS}

A metodologia abordada é um relato de experiência a partir de uma atividade proposta sobre função exponencial, elaborada com dados da bula do medicamento Diazepan, utilizando 
como recurso o software GeoGebra. Assim, elaborou-se um problema evidenciando como ocorre o processo de eliminação do Diazepan no organismo do paciente.

A partir do estudo na disciplina Tópicos de Cálculo do Programa de Pós-Graduação em Ensino de Ciências (PPGEC) da Universidade Estadual de Roraima - UERR, apresentouse uma proposta de atividade sobre função exponencial, elaborada com dados da bula do medicamento Diazepam, utilizando como recurso o software GeoGebra. Assim, elaborou-se um problema cujo objetivo é evidenciar como ocorre o processo de eliminação do Diazepam no organismo do paciente usuário.

O vídeo "Salvador - o hipocondríaco" subsidiou o ponto de partida das deduções levantadas na análise dos dados propostos pela bula do medicamento Diazepam. A bula deste medicamento descreve uma variação de possíveis reações e formas de ministrações específicas para cada caso e suas variações medicamentosas.

De acordo com a bula do Diazepam, o comprimido com $05 \mathrm{mg}$, de uso adulto e pediátrico, pertence a um grupo de medicamentos chamados benzodiazepínicos. Que é capaz de aliviar a ansiedade, a tensão nervosa e, em consequência, facilitar o sono. Além disso, produz relaxamento muscular e sua ação se faz cerca de 20 minutos após sua administração.

O Diazepam é facilmente absorvido após a administração oral e pode atingir a concentração plasmática máxima de 30 a 90 minutos.

Para a nossa pesquisa foi levantada a hipótese de que o pico em determinado paciente ocorreria após 60 minutos, e como a curva de eliminação do Diazepam é bifásica, uma fase de distribuição inicial com uma meia-vida que pode alcançar 03 horas e uma fase de eliminação terminal prolongada que pode alcançar 48 horas.

Como dose usual diária recomendadas adotamos $05 \mathrm{mg}$, embora existam casos que necessitem doses mais elevadas e usaremos como base a meia-vida de 03 horas.

Para que pudéssemos verificar o comportamento da eliminação do Diazepam no organismo de uma pessoa foi necessário utilizar o aplicativo Geogebra, sendo necessário plotar os pontos no gráfico que representam o decaimento e, a partir disso, utilizando a regressão exponencial, determinar a função que representa a situação hipotética.

Desse modo, o objetivo do nosso trabalho foi analisar as diferentes possibilidades na aplicação de Funções Exponenciais, identificar, construir e analisar o gráfico de uma função exponencial utilizando como fonte de recurso o Software Geogebra. 


\section{RESULTADOS E DISCUSSÃO}

\subsection{A Utilização do Software Geogebra no Conteúdo de Função Exponencial, Baseado na Teoria de Processos Mentais de Galperin.}

O planejamento do conteúdo de função exponencial foi realizado a partir da Teoria da Assimilação das Ações Mentais por Etapa proposta por Galperin, através do processo de internalização da atividade externa em interna que são: etapa motivacional, etapa boa e etapa da formação da ação no plano material.

Na Etapa Motivacional- constitui a etapa inicial, na qual não há nenhum tipo de ação a ser executada e tem como objetivo preparar os alunos para assimilarem os novos conhecimentos. Aqui foi apresentada a situação-problema e a proposta de utilizar os recursos oferecidos pelo Software GeoGebra para auxiliar na resolução do mesmo.

Na Etapa Base Orientadora da Ação - BOA- constitui o modelo da atividade, o projeto de ação, situando-se entre o sujeito e o objeto da ação, tendo como principal objetivo medir a relação entre a ação e a resolução da situação-problema.

Nessa etapa foi realizado um diagnóstico dos conhecimentos prévios dos acadêmicos de forma que pudesse ser observado as seguintes questões: O aluno está motivado? Está atento ás orientações? Participa da aula? A partir das orientações será necessário trabalhar as questões exploratórias: Que conceitos da matemática estão envolvidos no problema? Quais são as condições e limitações do problema? O problema tem incógnitas? Quais operações matemáticas são necessárias para resolver as questões?

Esse foi um momento crucial para o desenvolvimento dos trabalhos, tendo em vista que o aluno deve se apropriar das ferramentas do programa software GeoGebra para assim, entender como aplicá-lo e de que forma o software pode ajudar a encontrar a solução para a situação-problema.

Na Etapa de Formação da Ação no Plano Material ou Materializado -: nessa etapa o aluno começa a realizar a ação no plano externo, de forma detalhada. $\mathrm{O}$ aluno deve realizar as ações utilizando como apoio os esquemas da BOA e o professor tem a tarefa de controlar o cumprimento de cada uma das operações.

Portanto nessa etapa foi verificado se os alunos conseguem resolver a situação problema e se seguem as ações e operações da resolução de problemas de maneira correta.

A avaliação do aluno visa responder aos seguintes questionamentos: Como o aluno conseguiu desenvolver a atividade? Entendeu as orientações do docente? O aluno conseguiu 
interpretar as informações? Resolveu os problemas corretamente utilizando o modelo correto ensinado pelo professor? E por fim, encontrou a solução do problema?

A seguir estão expostos os passos percorridos para a resolução da situação-problema utilizando o Software Geogebra:

Inicialmente foi necessária a apropriação das ferramentas do programa Geogebra para o entendimento de como aplicá-lo e de que forma o software poderia ajudar a encontrar a solução para o problema em questão. A figura 1 mostra a janela de auto-ajuda do programa Geogebra:

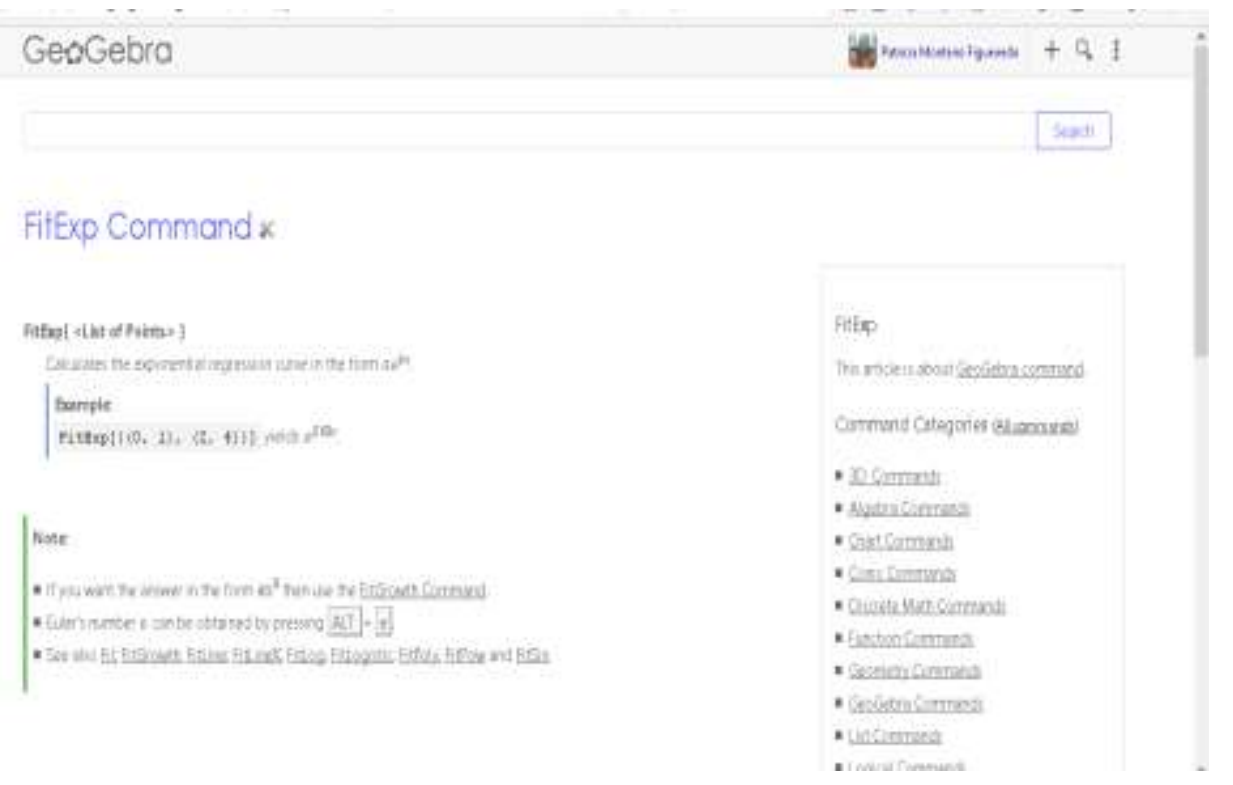

Figura 1: Janela de auto-ajuda do programa Geogebra. Fonte: Formatação on-line por cadastro pessoal.

Desse modo evidenciamos que se tratando de uma função exponencial, a curva deveria assumir o seguinte formato, como mostram a figura 2 : 


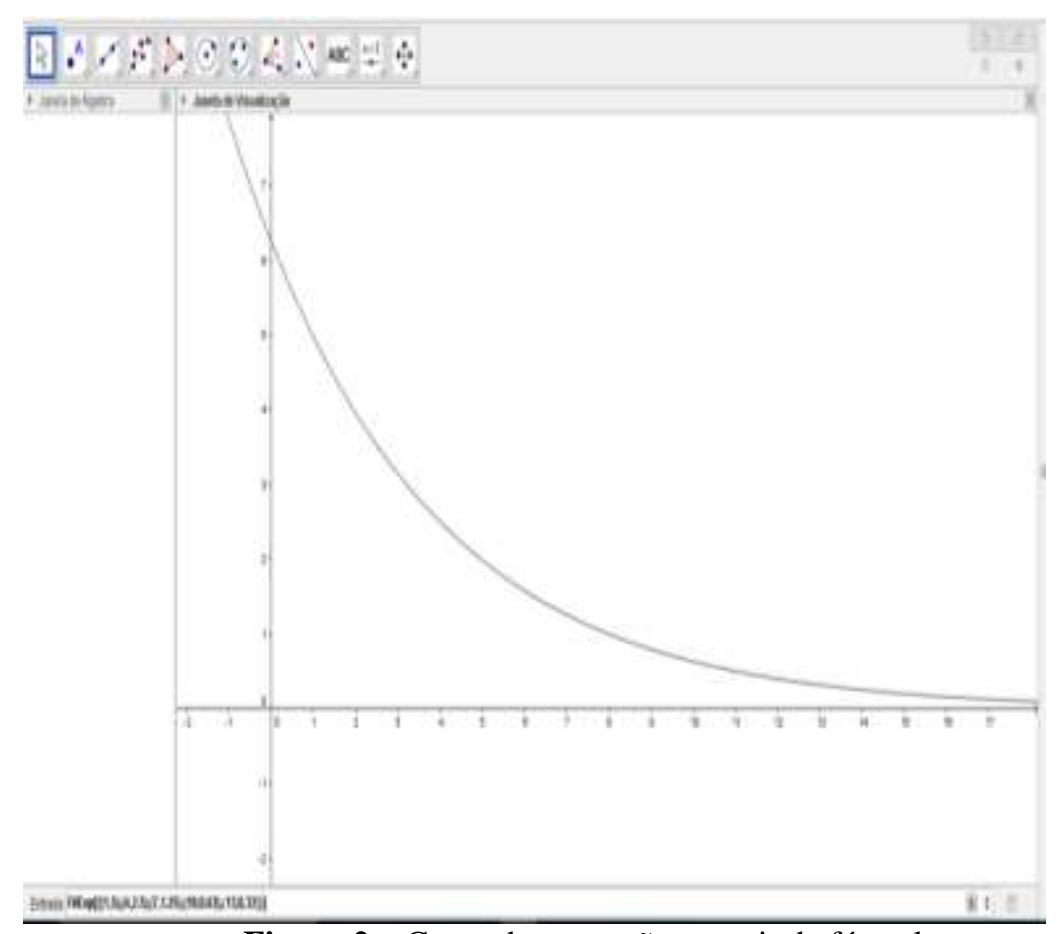

Figura 2 - Curva de regressão a partir da fórmula.

Fonte: Formatação on-line por cadastro pessoal através de login diferenciada.

E assim após aplicar a fórmula que correspondesse ao problema proposto no aplicativo Geogebra obteve-se o gráfico da função, como mostra na figura 3:

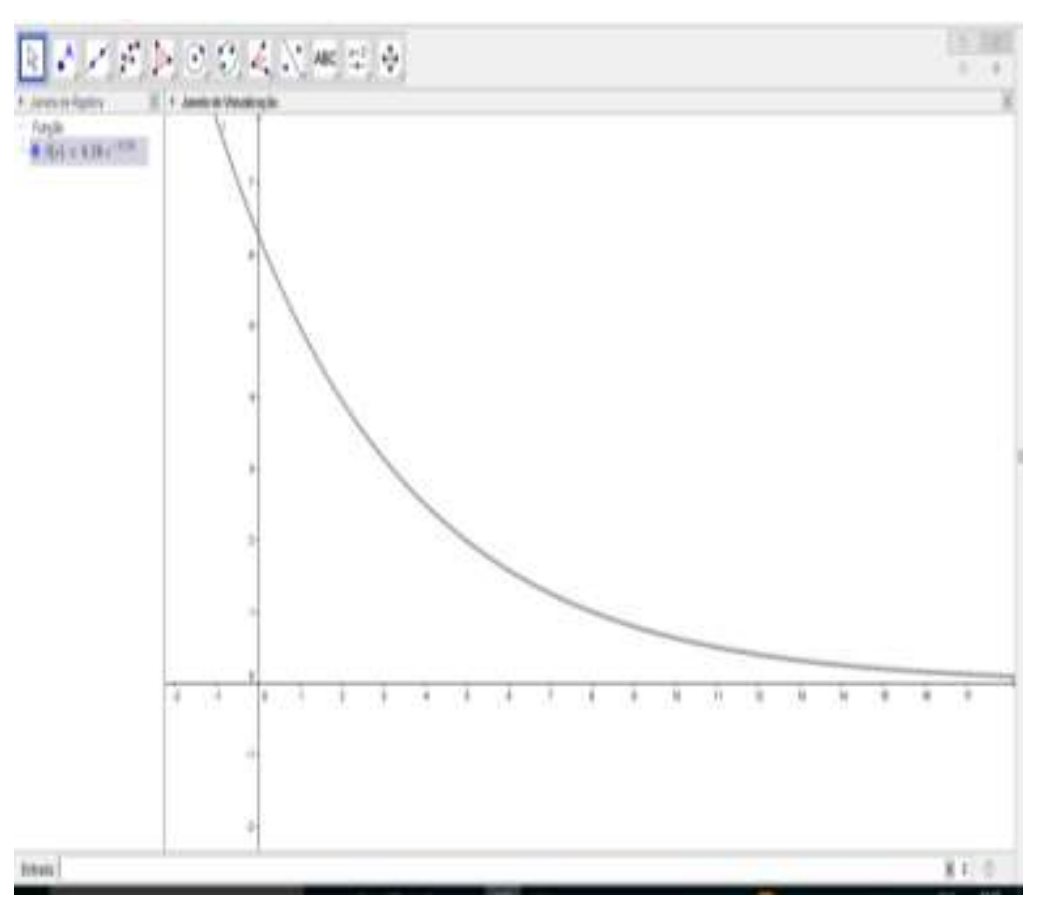

Figura 3 - Função exponencial do problema

Fonte: Formatação on-line por cadastro pessoal através de login diferenciada.

Durante todo o processo foi necessário utilizar o Geogebra on-line e fazer o cadastro para que pudéssemos chegar aos resultados necessários que explicitassem o resultado da 
pesquisa sobre o uso do Diazepam e suas administrações durante o pico e a meia-vida do medicamento dentro do corpo.

Na Etapa de Formação da Ação Verbal, externa-os elementos da ação são representados na forma verbal, não sendo mais utilizados apoios externos.

Será analisada a capacidade de argumentação e explicação do processo de resolução, esta verbalização pode ser de forma escrita ou oral. $\mathrm{O}$ aluno precisa ser avaliado pelas seguintes perguntas: Encontrou a solução do problema? Interpretou os resultados? Conseguiu explicar as ações e operações que utilizou durante o processo de resolução? E se este apresenta argumentos, ideias, opiniões de forma clara?

A partir do gráfico ilustrado na figura 4, pode-se observar a curva de regressão dos pontos A, B, C, D e E. Assim perceber o pico do uso do Diazepam e sua meia-vida durante a absorvição no organismo.

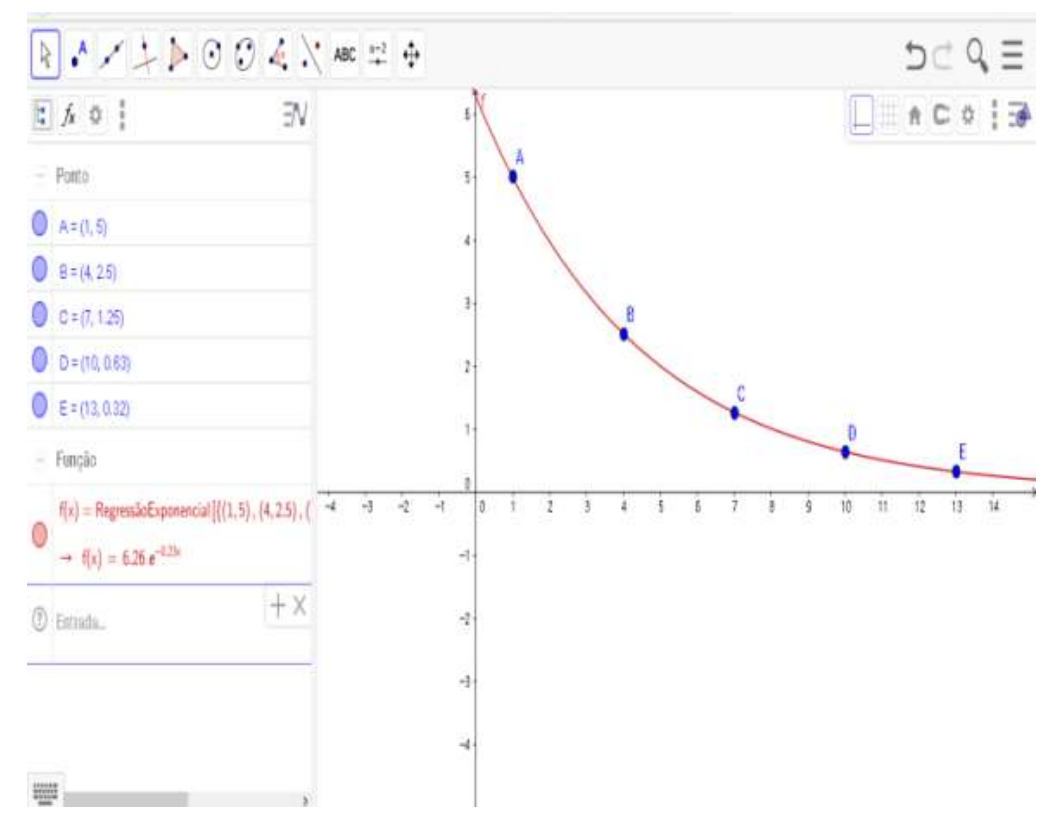

Figura 4 - Plotagem dos pontos A, B, C, D e E na curva de regressão. Fonte: Formatação on-line por cadastro pessoal através de login diferenciada.

Após análise dos dados aplicados no GeoGebra para determinar a função exponencial que atenda ao problema que foi levantado, uma vez que a meia - vida do medicamento Diazepam no organismo do ser humano é de 03h, foi possível perceber, através do decaimento da curva, que conforme o tempo aumenta, a quantidade de medicamento diminui. Isso ocorre rapidamente até os primeiros 20 minutos que chamamos de "pico" e em seguida, de três em três horas, cai pela metade até sua total absorção pelo organismo. 


\section{CONSIDERAÇÕES}

A resolução de problemas aliada as TD tem sido tratada como uma tendência metodológica para fundamentar a prática do ensino da matemática.

Tendo como base a situação problema proposta, partimos do pressuposto de que o paciente necessita apenas de $05 \mathrm{mg}$ da substancia por dia e suas possíveis variações de pico e tempo meia-vida expressos na função exponencial e sua aplicabilidade usando como fonte de recurso o programa GeoGebra.

Após análise dos dados aplicados no GeoGebra para determinar a função exponencial que atenda ao problema que foi levantado, uma vez que a meia - vida do medicamento Diazepam no organismo do ser humano é de 03h, foi possível perceber, através do decaimento da curva, que conforme o tempo aumenta, a quantidade de medicamento diminui. Isso ocorre rapidamente até os primeiros 20 minutos que chamamos de "pico" e em seguida, de três em três horas, cai pela metade até sua total absorção pelo organismo.

Podemos observar que as etapas propostas por Galperin observadas nesse trabalho foram alcançadas até a Etapa de Formação da Ação Verbal Externa, pois é nessa etapa que é analisada a capacidade de argumentação e explicação do processo de resolução. Esta verbalização foi feita de forma escrita a partir da observação do gráfico que demonstra o decaimento da curva e assim determinou a função exponencial que atenda ao problema.

Assim, evidenciou-se que os recursos disponibilizados no software GeoGebra contribuem para a resolução de problemas que envolvem a aplicação de Função Exponencial, pois, assim como Borba, Silva e Gadanidis (2014, p. 63), compreende-se que "[...] uma exploração acerca das potencialidades do GeoGebra nos levaram a considerar uma construção diferenciada para explorar a noção de Função Exponencial”.

\section{REFERÊNCIAS}

BORBA, M. C.; SCUCUGLIA, R.; GADANIDIS, G. (2014). Fases das tecnologias digitais em educação matemática: sala de aula e internet em movimento. São Paulo.

DANTE, L. R. Didática da resolução de problemas de Matemática. $1^{\text {a }}$ a $5^{\text {a }}$ Série. $12^{\mathrm{a}}$ ed. Ática 2000.

FREIRE, A. A. C. O uso do Geogebra na resolução de problemas matemáticos a a partir da teoria de Galperin. Boa Vista/RR: UERR,2016.

MICHELS, J. O processo ensino aprendizagem da tabuada: Desvendando práticas e criando possibilidades. Criciúma, 2009. 
MUSSATO, S. Cyber formação com professores de matemática à distância: horizontes que emergem de diferentes contextos culturais/ Solange Mussato. - Canoas, 2015.

PCN. Parâmetros curriculares nacionais: matemática/ Ministério da Educação. Secretaria de Educação Fundamental. -3. ed.- Brasília: A Secretaria, 2001.

PERIUS, A. A. B., A Tecnologia Aliada ao Ensino de Matemática Cerro Largo/RS, 2012. Disponível: < https://pt.scribd.com/document/327063163/A-tecnologia-aliada-ao-ensino-damatematica-pdf>. Acesso em: 19 de novembro de 2018.

STAREPRAVO, A. R. Mundo das ideias: jogando com a matemática, número e operações / Ana Ruth Starepravo. Curitiba: ed. Aymará, 2009.

Submetido em: 16 de janeiro de 2020 .

Aprovado em: 14 de maio de 2020. 